\title{
VARIABILIDADE DE MECANISMOS NA APRENDIZAGEM INDIVIDUAL SOBRE SOFTWARE LIVRE ${ }^{1}$
}

\author{
Cláudio Bezerra Leopoldino ${ }^{2}$ \\ Elisabeth Regina Loiola da Cruz Souza ${ }^{3}$
}

http://dx.doi.org/10.1590/1413-2311.151.54506

\begin{abstract}
RESUMO
Dentre os diversos aspectos da aprendizagem individual nas organizações, a variabilidade dos mecanismos de aprendizagem empregados é um dos mais importantes. Estudos sobre o assunto afirmam que a utilização de uma maior variedade de mecanismos de aprendizagem individual proporcionaria uma aprendizagem mais efetiva. No entanto, esta visão não é unânime, pois há questionamentos da relação entre a variedade de mecanismos e maior aprendizagem, o que serve como estímulo a novas investigações. O presente artigo apresenta os mecanismos de aprendizagem individual mais significativos identificados na Superintendência de Desenvolvimento de Sistemas (Supde) de uma empresa pública de tecnologia da informação vivenciados na aquisição de conhecimentos sobre softwares livres, e o levantamento da sua variabilidade. A investigação dos mecanismos de aprendizagem individual que são empregados em uma organização é relevante por duas grandes razões: para identificar os mecanismos de aprendizagem individual mais significativos e como forma de eliminar de futuras análises os mecanismos de aprendizagem individual que não apresentem real importância para população estudada. Não foi encontrada associação estatística entre maior variabilidade dos mecanismos de aprendizagem adotados e a aprendizagem obtida, o que gerou novos questionamentos sobre esta relação. A pesquisa abordou tanto as modalidades de aprendizagem individual formal quanto informal.
\end{abstract}

Palavras-Chave: Aprendizagem Individual. Software Livre. Variabilidade da Aprendizagem Individual.

\section{VARIABILITY MECHANISMS IN INDIVIDUAL LEARNING ABOUT FREE OPEN SOURCE SOFTWARE}

\begin{abstract}
Among the various aspects of individual learning in organizations, the variability of employees learning mechanisms is one of the most important issues. Studies on the subject stated that the use of a greater variety of individual learning mechanisms provide a more

\footnotetext{
${ }^{1}$ Recebido em 26/03/2015; aprovado em 26/06/2017.

${ }^{2}$ Universidade Federal do Ceará - claudio.leopoldino@ufc.br.

${ }^{3}$ Universidade Federal da Bahia - beloi@ufba.br.
} 
effective learning. However, this view is not unanimous, as there is questioning of the relationship between the variety of mechanisms and higher learning, which serves as a stimulus to further research. This article presents the most significant individual learning mechanisms identified in the Systems Development Department of a public company of information technology, in the acquisition of knowledge about free open source software, and the assessment of its variability. The investigation of the individual learning mechanisms that are employed in an organization is relevant for two main reasons: to identify the most significant individual learning mechanisms and as a way of eliminating from future analyzes the individual learning mechanisms that have no real relevance for population's study. There was no significant association between greater variability of the adopted mechanisms of learning and the learning obtained, which generated new questions about this relationship. The research addresses the formal individual learning and the informal individual learning.

Keywords: Individual Learning. Free Open Source Software. Variability of Individual Learning.

\section{VARIABILIDAD EN MECANISMOS DE APRENDIZAJE INDIVIDUAL ACERCA DE SOFTWARE LIBRE}

\section{RESUMEN}

Entre los diversos aspectos del aprendizaje individual en las organizaciones, la variabilidad de los mecanismos de aprendizaje es uno de los temas más importantes. Los estudios sobre el tema indicaron que el uso de una mayor variedad de mecanismos de aprendizaje individuales proporciona un aprendizaje más eficaz. Sin embargo, esta opinión no es unánime, ya que hay cuestionamiento de la relación entre la variedad de mecanismos y educación superior, que sirve de estímulo para futuras investigaciones. Este artículo presenta los mecanismos de aprendizaje individual más significativos identificados en el desarrollo de sistemas de una empresa pública para la tecnología de la información, con experiencia en la adquisición de conocimientos sobre el software libre, y el levantamiento de su variabilidad. La investigación de los mecanismos de aprendizaje individual empleados en una organización muestra a ser relevante por dos razones principales: identificar los mecanismos de aprendizaje individuales más importantes y como una forma de eliminar los análisis futuros mecanismos de aprendizaje individuales que no tienen verdadera relevancia para la población del estudio. No se encontró asociación significativa entre una mayor variabilidad de los mecanismos adoptados de aprendizaje y el aprendizaje obtenido, lo que generó nuevas preguntas acerca de esta relación. El estudio abordó tanto el aprendizaje individual formal como el aprendizaje individual informal.

Palabras clave: aprendizaje individual. Software Libre. Variabilidad de aprendizaje individual.

\section{INTRODUÇÃO}

A capacidade de aprender e aplicar os conhecimentos adquiridos é uma necessidade cada vez mais importante nas organizações. O surgimento de novas leis, processos, materiais,

REAd | Porto Alegre - Vol. 23 - No Especial - Dezembro 2017 - p. 210-231 
ferramentas, concorrentes e demandas da sociedade altera o ambiente, exigindo adaptações organizacionais que se refletem na necessidade dos seus integrantes de adquirir e empregar novos conhecimentos, habilidades e atitudes. Pode-se afirmar que a necessidade de aprender é uma constante no mundo corporativo, e que a aprendizagem, em seus vários aspectos, tornase cada vez mais um facilitador do rendimento da organização (BELL; MENGÜÇ; WIDING II, 2009; LÓPEZ, 2006; FIGUEIREDO, 2006; CASTRO; FIGUEIREDO, 2005; TACLA; FIGUEIREDO, 2003). A aprendizagem individual encontra neste cenário dinâmico um ambiente propício para se desenvolver.

Dentre os diversos aspectos da aprendizagem individual nas organizações, a variabilidade dos mecanismos de aprendizagem empregados é um dos mais importantes (COOK et al., 2010). A posição predominante afirma que a utilização de uma maior variedade de mecanismos de aprendizagem individual proporcionaria uma maior aprendizagem (FIGUEIREDO, 2006; ROSAL; FIGUEIREDO, 2006; CASTRO; FIGUEIREDO, 2005; TACLA; FIGUEIREDO, 2003). No entanto, esta visão não é unânime em todos os estudos, pois há questionamento da relação entre a variedade de mecanismos e maior aprendizagem (LOIOLA; PEREIRA; GONDIM, 2011; LOIOLA; PORTO, 2008; LOIOLA, 2007; NERIS, 2005). Esta divergência não se resolverá sem que existam mais estudos que abordem a variedade dos mecanismos de aprendizagem nas organizações.

Tendo como motivação a lacuna teórica na literatura, buscou-se fazer o levantamento dos mecanismos de aprendizagem utilizados em uma organização, para em seguida abordar a sua variabilidade. $\mathrm{O}$ presente artigo apresenta os mecanismos de aprendizagem individual mais significativos identificados na Superintendência de Desenvolvimento de Sistemas (Supde) de uma empresa pública de tecnologia da informação, vivenciadas na aquisição de conhecimentos sobre softwares livres. Adicionalmente, foi feito levantamento da variabilidade das formas pelas quais os funcionários aprendem sobre as tecnologias supracitadas na organização. Por fim, foi testada a relação estatística entre variabilidade de mecanismos de aprendizagem e o conhecimento efetivamente obtido pelos aprendizes. A pesquisa abordou tanto os mecanismos de aprendizagem individual formal quanto informal.

$\mathrm{O}$ artigo inicia por esta introdução. Em seguida, é apresentada a pesquisa bibliográfica realizada sobre a variabilidade nos mecanismos de aprendizagem individual formal e informal. Seguem a metodologia, os resultados obtidos e sua discussão, e as notas conclusivas. 


\section{APRENDIZAGEM INDIVIDUAL NAS ORGANIZAÇÕES E A SUA VARIABILIDADE}

A principal característica da aprendizagem individual é o fato de ser o resultado de um processo cognitivo interno aos seres humanos, consistindo em fenômeno que ocorre "dentro da cabeça" de quem aprende (SIMON, 1991). Adicionalmente, afirma-se que a aprendizagem individual ocorre pela interação entre as pessoas e do aprendiz com o meio (COELHO JÚNIOR; BORGES-ANDRADE, 2008; LOIOLA; PORTO, 2008). A revisão bibliográfica desta pesquisa sobrelevou duas importantes formas de aprendizagem individual nas organizações: a aprendizagem individual formal e a informal.

A aprendizagem formal é planejada, sistematizada e direcionada para um determinado fim (LAI; KHADDAGE; KNEZEK, 2013). Estruturada e oferecida por uma instituição, que pode ser ou não a empresa onde trabalha o aprendiz, apresenta determinado tipo de conhecimento/ habilidade de forma padronizada e organizada aos indivíduos, visando à sua aquisição e à sua aplicação de forma facilmente reproduzível e, ao mesmo tempo, adequada às necessidades. Essa uniformização pressupõe a definição de métodos, técnicas, lugares e condições específicas prévias que são criadas deliberadamente para suscitar ideias, conhecimentos, valores, atitudes e comportamentos (COELHO JÚNIOR; BORGESANDRADE, 2008). Apresenta, como principal benefício, a otimização dos processos de aquisição, retenção e generalização dos comportamentos, habilidades e atitudes (D'AMELIO, 2007).

A aprendizagem informal, por sua vez, não depende do planejamento preparatório ou controle da organização de trabalho ou de uma instituição de ensino. Não existe na aprendizagem informal uma programação e organização prévia de conhecimentos e habilidades a serem apresentados aos aprendizes (COELHO JÚNIOR; BORGESANDRADE, 2008; WILLE, 2005; ERAUT, 2004). A aprendizagem informal apresenta maior sutileza e não conta com objetivos, métodos e recursos definidos sistematicamente com antecedência. É predominantemente não institucional e experimental (CONLON, 2004; ERAUT, 2004). Não está atrelada a um tutor; não demanda planejamento prévio; tem, geralmente, aplicabilidade e transferência imediata; não há controle da organização acerca do que é aprendido, podendo estar ou não atrelada aos objetivos organizacionais ou a algum tipo de qualificação (LAI; KHADDAGE; KNEZEK, 2013; PANTOJA; BORGES-ANDRADE, 2009, COELHO JÚNIOR; BORGES-ANDRADE, 2008; ERAUT, 2004).

REAd | Porto Alegre - Vol. 23 - No Especial - Dezembro 2017 - p. 210-231 
Tanto a aprendizagem formal quanto a informal são importantes para as organizações, não se recomendando o predomínio de uma modalidade sobre a outra (CZERKAWSKI, 2016; COELHO JÚNIOR; BORGES-ANDRADE, 2008; D'AMELIO, 2007; WILLE, 2005). D'Amelio (2007) afirma, com base em pesquisa realizada em organização pública no Brasil, que tanto a aprendizagem formal quanto a informal dos indivíduos são significativas e podem ocorrer de forma integrada assim como em separado. Lai, Khaddage e Knezek (2013) afirmam ser possível integrar estas duas aprendizagens por meio de ferramentas online colaborativas, de comunicação e de coordenação. Tanto as redes formais quanto as informais são utilizadas por professores e estudantes em seus processos de aprendizagem (CZERKAWSKI, 2016; BULL et al., 2008).

A literatura pesquisada indica relação entre maiores graus de variabilidade de mecanismos de aprendizagem e maior aprendizagem ocasionada por meio de um maior fluxo de conhecimentos (FIGUEIREDO, 2006; CASTRO; FIGUEIREDO, 2005; TACLA; FIGUEIREDO, 2003), posição não confirmada em todos os estudos (LOIOLA; PEREIRA; GONDIM, 2011). Também foi constatada, em outras investigações, uma baixa variabilidade de processos de aprendizagem nas organizações pesquisadas (LIMA; DE LIMA; CABRAL, 2013; LOIOLA; PEREIRA; GONDIM, 2011; LOIOLA; PORTO, 2008; LOIOLA, 2007). Ainda que a relação entre a variabilidade de mecanismos de aprendizagem individual e a aprendizagem tenha se mostrado efetiva, o número de mecanismos de aprendizagem empregados pela população pode ser pequeno.

\section{METODOLOGIA}

O estudo apresentou características quantitativas e a coleta de dados foi realizada por meio de survey. O questionário foi elaborado com base em revisão bibliográfica (SOUZA, 2007; D'AMELIO, 2007; CORREIA, 2007; NERIS, 2005; LEOPOLDINO, 2004; TACLA; FIGUEIREDO, 2003). A lista de itens relativos aos mecanismos de aprendizagem formais e informais foi obtida de D'Amelio (2007) e Correia (2007).

Sua validação contou com duas etapas de pré-testes e um teste piloto, visando o aprimoramento do instrumento e a sua validação. A coleta de dados via internet foi definida como meio para a obtenção de dados.

O instrumento de coleta, em sua versão definitiva, apresentou inicialmente indagação sobre o software livre mais utilizado, mostrando ao respondente uma lista para seleção. Com 
base no software selecionado, o entrevistado deveria descrever o grau de aprendizagem sobre a tecnologia nos últimos dois anos e indicar a importância dos mecanismos de aprendizagem individual que foram utilizados para aquisição de conhecimento sobre a ferramenta selecionada. Foi empregada Escala Likert de 1 a 5, apresentando as opções: "Não se aplica"; "Nenhuma Importância"; "Baixa"; "Moderada"; "Alta" e "Importância Muito Alta".

Para delimitar a área de conhecimento sobre a qual seriam feitos os questionamentos, foi escolhido o escopo da aprendizagem sobre softwares livres aplicados no ambiente de trabalho. A facilidade de distribuição e utilização, a variedade de ferramentas oferecidas e o ritmo das atualizações faz com que haja um processo constante de renovação e aprendizagem por parte dos integrantes destas comunidades, auxiliada pela interação em rede, o que motivou a definição deste delimitador (SINGH; YOUN; TAN, 2010; CORREIA, 2007; ROCHA, 2007). A coleta foi informatizada por meio de sítio projetado pelos pesquisadores e divulgado dentro de uma grande empresa pública de tecnologia da informação. A organização pesquisada apresenta um grande número de funcionários, comunidades, projetos em andamento, sistemas e soluções total ou parcialmente desenvolvidas em software livre. $\mathrm{O}$ fato de ser uma empresa pública foi um estímulo adicional à pesquisa, uma vez que a literatura de pesquisa em aprendizagem apresenta foco em instituições privadas (RASHMAN; WITHERS; HARTLEY, 2009; ANTONELLO; GODOY, 2009).

A pesquisa foi realizada sobre uma população estimada em 2500 indivíduos, da Superintendência de Desenvolvimento da Empresa (Supde). Trata-se de um quadro funcional relativamente jovem, com forte predominância de desenvolvedores de sistemas que tiveram acesso ao ensino superior e à pós-graduação e que apresentam um bom nível de experiência dentro e fora da empresa, tendo participado de um número substancial de projetos de desenvolvimento de software. Houve considerável renovação dos integrantes da Supde através de concursos realizados pouco tempo antes da coleta de dados.

Obtiveram-se 256 questionários válidos de 340 ao todo. Foram removidos da análise questionários com preenchimento incompleto e vícios de preenchimento. Dos dados dos indivíduos pode-se traçar um perfil do respondente padrão: sexo masculino (83,2\%), de 25 a $35 \operatorname{anos}(56,3 \%)$, exercendo função técnica de desenvolvimento $(89,1 \%)$. Quanto à formação, o funcionário da Supde em geral apresenta nível superior (mais de 96\%), sendo que deste montante o número de indivíduos com pós-graduação supera o dos que possuem apenas a graduação (40,2\% fizeram especialização e 11,3\% concluíram um mestrado). Quanto à experiência dos respondentes, apenas $20,7 \%$ possuíam 5 anos ou menos de experiência, e a 
média de experiência é de 12,17 anos. O tempo de empresa 7,17 anos e tendo participado de 10 a 49 projetos $(44,3 \%)$.

A análise dos dados compreendeu análise estatística descritiva, testes t para cálculo de diferença de médias, histogramas e análise de correlações.

\section{RESULTADOS}

Os dados obtidos permitiram uma visão ampla das tecnologias livres mais utilizadas, do grau de aprendizagem obtido e dos mecanismos de aprendizagem individual formal e informal mais frequentes que caracterizam o grau de variabilidade dos processos de aprendizagem efetivamente empregados na organização.

\subsection{TECNOLOGIAS APRENDIDAS}

A escolha das ferramentas por parte dos respondentes mostrou forte relação entre as tecnologias escolhidas e as atividades desempenhadas no trabalho. Os softwares da lista aparecem, com uma breve descrição, na Tabela 1, na qual são destacadas em cinza as tecnologias que tiveram mais de 15 citações.

Destacaram-se a linguagem de programação Java, a interface de desenvolvimento Eclipse, a ferramenta de gestão de configuração de software CVS, o gerenciador de e-mails Expresso, software livre desenvolvido na própria empresa, o sistema operacional Linux, o navegador web Firefox e o conjunto de ferramentas para escritório BrOffice/ LibreOffice. Todas estas ferramentas são adotadas institucionalmente pela empresa, embora não sejam obrigatórias para todos os projetos, em virtude das demandas dos clientes e das centenas de sistemas legados com tecnologias proprietárias e livres variadas. Ferramentas mais específicas como o Testlink, utilizado em testes de software, tiveram menor grau de utilização e consequentemente foram menos escolhidas pelos respondentes. Apenas 5 respondentes forneceram outras ferramentas que não constavam da lista, e deste conjunto de respostas apenas a ferramenta livre PostgreSQL foi citada mais de uma vez. Outras ferramentas mencionadas foram o Framework Qt / C++ e o pl/sql developer.

A linguagem de programação Java foi a tecnologia mais citada, uma vez que é utilizada em diversos projetos da empresa. A ferramenta Eclipse foi a interface de programação mais citada, sendo muitas vezes utilizada em conjunto com tecnologia Java ou 
CVS. O CVS é uma ferramenta largamente utilizada internamente como repositório de artefatos de desenvolvimento, permitindo o adequado versionamento e a sua integridade.

Tabela 1 - Ferramentas escolhidas pelos respondentes

\begin{tabular}{|c|c|c|}
\hline Ferramenta & Descrição & Citações \\
\hline Apache (Servidor Web) & Servidor web & 1 resposta $-0,4 \%$ \\
\hline Apache TomCat & Servidor de aplicações & $2-0,8 \%$ \\
\hline BrOffice/ LibreOffice & $\begin{array}{l}\text { Automação de escritórios (planilha, } \\
\text { editor de texto, etc.) }\end{array}$ & $21-8,2 \%$ \\
\hline CVS & $\begin{array}{l}\text { Gerência de configuração de } \\
\text { software. }\end{array}$ & $34-13,3 \%$ \\
\hline Expresso & Gerenciador de E-mails & $27-10,5 \%$ \\
\hline Firefox & Navegador web & $19-7,4 \%$ \\
\hline Eclipse & $\begin{array}{l}\text { Interface de Desenvolvimento } \\
\text { (IDE) }\end{array}$ & $38-14,8 \%$ \\
\hline Java & Linguagem de programação & $55-21,5 \%$ \\
\hline Java Demoiselle & $\begin{array}{l}\text { Framework para Desenvolvimento } \\
\text { Java }\end{array}$ & $9-3,5 \%$ \\
\hline Linux & Sistema Operacional Livre & $22-8,6 \%$ \\
\hline Mantis & $\begin{array}{c}\text { Ferramenta de Registro de Bugs/ } \\
\text { Ocorrências }\end{array}$ & $7-2,7 \%$ \\
\hline PHP & Linguagem de programação & $7-2,7 \%$ \\
\hline Python & Linguagem de programação & $2-0,8 \%$ \\
\hline Testlink & $\begin{array}{l}\text { Ferramenta de auxílio a testes de } \\
\text { software. }\end{array}$ & $7-2,7 \%$ \\
\hline
\end{tabular}

A citação do navegador web Firefox e do gerenciador de correio eletrônico Expresso mostram que muitos dos respondentes exploram com frequência estes softwares cotidianamente, utilizando a intranet e a internet no trabalho para comunicação, visualização de conteúdo laboral e utilizando os sistemas organizacionais. O destaque dado ao Linux se deve ao processo de migração de estações de trabalho para software livre, que fez com que a base instalada na empresa deste software abrangesse boa parte da área de desenvolvimento. $\mathrm{O}$ BrOffice/ LibreOffice foi bastante citado pela sua robustez e versatilidade, e é utilizado como meio de geração e visualização de documentos corporativos para uso interno e externo.

\subsection{APRENDIZAGEM DOS INDIVÍDUOS}

Os respondentes também foram questionados a respeito da aprendizagem obtida na ferramenta selecionada nos últimos dois anos. Em geral os respondentes passaram de REAd | Porto Alegre - Vol. 23 - No Especial - Dezembro 2017 - p. 210-231 
conhecimento Baixo/ Moderado (média 2,7550) a Moderado/ Alto (média 3,5191). A diferença de aprendizagem nos dois anos foi de 0,8263, um pouco abaixo de um nível de conhecimento na escala adotada. $\mathrm{O}$ cálculo da aprendizagem individual pela subtração entre o nível de conhecimento atual e nos dois anos anteriores indica o quanto de conhecimento foi adquirido e retido no período, podendo ser aplicado, generalizado e transferido.

A Tabela 2 apresenta as estatísticas descritivas do conhecimento sobre a ferramenta livre há dois anos, do conhecimento atual e do conhecimento obtido, calculado pela diferença entre o conhecimento atual e o anterior.

Tabela 2 - Aprendizagem individual - estatísticas descritivas

\begin{tabular}{lccccccc}
\hline & N & Intervalo & Mínimo & Máximo & Média & $\begin{array}{l}\text { Desvio } \\
\text { Padrão }\end{array}$ & Variância \\
\hline $\begin{array}{l}\text { Conhecimento na } \\
\text { ferramenta há } \\
\text { dois anos }\end{array}$ & 249 & 4,00 & 1,00 & 5,00 & 2,7550 & 1,08890 & 1,186 \\
$\begin{array}{l}\text { Conhecimento na } \\
\text { ferramenta } \\
\text { atualmente }\end{array}$ & 235 & 3,00 & 2,00 & 5,00 & 3,5191 &, 74152 &, 550 \\
$\begin{array}{l}\text { Conhecimento } \\
\text { obtido }\end{array}$ & 236 & 5,00 & $-1,00$ & 4,00 &, 8263 &, 92225 &, 851 \\
\hline
\end{tabular}

Fonte: Dados da pesquisa.

A aprendizagem individual também ocorreu de forma negativa em algumas situações. Sete respondentes (3\% das respostas válidas) relataram aprendizagem negativa, ou seja, a perda de parte do domínio sobre a tecnologia livre no período. Existe a possibilidade de parte de esse grupo ter obtido um bom nível conhecimento nas ferramentas selecionadas, mas não ter recebido a oportunidade de aplicá-lo e mantê-lo atualizado nos últimos dois anos, ficando defasado pela evolução da tecnologia selecionada e pela falta de prática que afetou negativamente os processos de aquisição e retenção.

Um número substancial dos respondentes (92, cerca de 39\% das respostas válidas) não apresentou diferenças no nível de conhecimento nos últimos dois anos, o que aparentemente foi causado por vários fatores: em alguns casos, o conhecimento obtido atingiu um nível satisfatório e se estabilizou no tempo; em outras situações a ferramenta escolhida não apresentou evolução substancial, o que restringiu a aprendizagem, e em certos casos os usuários já apresentavam um nível de conhecimento alto nos últimos dois anos, o que fez com que não houvesse margem suficiente para evolução significativa.

A maioria dos respondentes $(58,1 \%$ das respostas válidas) relatou aumento na aprendizagem sobre a ferramenta nos últimos dois anos. A aplicação de teste t revelou que as REAd | Porto Alegre - Vol. 23 - No Especial - Dezembro 2017 - p. 210-231 
variáveis "Conhecimento na ferramenta há dois anos" e "Conhecimento na ferramenta atualmente" diferem entre si de forma estatisticamente significativa $(\mathrm{t}(230)=13,674, \mathrm{p}<.001)$, com o conhecimento atual sendo superior ao anterior. Este resultado mostra que na Supde houve um ganho de conhecimento estatisticamente significativo sobre as ferramentas utilizadas por parte dos indivíduos, embora, em muitos casos, o conhecimento tenha se estabilizado e os indivíduos tenham mantido seus níveis de conhecimento sem progresso significativo, mas se mantendo atualizados com a tecnologia.

\subsection{APRENDIZAGEM FORMAL}

Foi realizado teste t para identificar as variáveis que são efetivamente significativas para a aprendizagem, de modo similar ao procedimento descrito por Loiola, Pereira e Gondim (2011) e Correia (2007). O critério utilizado visou destacar as variáveis cuja média fosse estatisticamente maior que 3, o valor central da escala, empregando o teste t para uma única amostra. A análise dos itens relativos à aprendizagem formal empregando o teste t para uma única amostra revelou que nenhum dos itens obteve média superior ao ponto de corte. Não foi identificado nenhum item da aprendizagem individual formal que apresentasse portanto uma relevância significativa, situação já ocorrida nos pré-testes do instrumento de coleta. Este resultado indica que os respondentes, apesar de terem acesso a múltiplas opções de mecanismos de aprendizagem formal, não atribuíram a estes um alto grau de importância para o ganho de conhecimento sobre a ferramenta livre escolhida. A Tabela 3 mostra o valor de N, as médias e o teste $\mathrm{t}$ realizado.

Os itens relativos a treinamentos, "Curso(s)/ treinamento(s) de curta duração (até 40h)" e "Palestra(s)", obtiveram os maiores valores para o N, indicador da relevância da aprendizagem formal por treinamentos formais de curta e longa duração e de palestras para a aprendizagem sobre tecnologias livres na Supde. Estes resultados corroboram os resultados de pesquisas que indicam impacto de treinamentos tanto em profundidade quanto na abrangência da aprendizagem (ZERBINI, 2007; HANKE, 2006; ARAÚJO, 2005).

Uma boa parcela da menor importância dos itens da aprendizagem individual formal pode ser explicada em parte por características do ambiente da empresa.

No caso da aprendizagem pela "Participação em grupos de estudo formais", observase que a formação de grupos de estudo formais relativos a ferramentas de software é pouco frequente na Supde. $\mathrm{O}$ mecanismo de aprendizagem relativa à participação em congressos e 
outros eventos relativos a ferramentas de software livre não foi mais efetivo por ser pouco utilizado. Custos como a inscrição em eventos, passagens e hospedagem, aportes financeiros ou liberação da jornada de trabalho por parte da empresa, não são ressarcidos aos possíveis aprendizes, salvo em casos excepcionais em que seja claro o retorno do investimento à organização.

No caso do mecanismo "Preparação para certificação", a baixa importância ocorre em virtude da maioria das ferramentas de software livre não apresentar programas estruturados de certificação, o que inclui ferramentas como Postgresql e Testlink, e fez com que a variável obtivesse o menor $\mathrm{N}$ entre os mecanismos de aprendizagem formal. Atualmente existem vários softwares livres que apresentam programas de certificação com avaliações rigorosas que conferem aos profissionais uma qualificação certificada pela comunidade, ou por alguma grande empresa ou instituição, como o Linux. No entanto, esta não é uma realidade para a maioria das demais ferramentas. Adicionalmente, no caso dos softwares livres desenvolvidos na empresa, não existe certificação individual para os usuários. Neste caso, a formação é feita por meio de treinamentos realizados de acordo com a necessidade, dentro e fora da empresa, para clientes externos e internos.

Tabela 3 - Aprendizagem individual formal

\begin{tabular}{|c|c|c|c|c|c|c|c|c|}
\hline & \multirow[t]{2}{*}{$\mathbf{N}$} & \multirow[t]{2}{*}{ Média } & \multirow[t]{2}{*}{$\mathbf{t}$} & \multirow[t]{2}{*}{$\begin{array}{l}\text { Graus } \\
\text { de lib. }\end{array}$} & \multirow[t]{2}{*}{ Sig } & \multirow[t]{2}{*}{$\begin{array}{l}\text { Dif. de } \\
\text { média }\end{array}$} & \multicolumn{2}{|c|}{$\begin{array}{c}\text { Int. de conf. de } \\
95 \%\end{array}$} \\
\hline & & & & & & & mínimo & máximo \\
\hline $\begin{array}{l}\text { Participação em grupos de } \\
\text { estudo formais }\end{array}$ & 146 & 2,0753 & $-10,697$ & 145 & $*$ &,- 92466 & $-1,0955$ &,- 7538 \\
\hline $\begin{array}{l}\text { Curso(s)/ treinamento(s) de } \\
\text { curta duração (até } 40 \mathrm{~h})\end{array}$ & 201 & 2,9801 &,- 233 & 200 &, 816 &,- 01990 &,- 1885 &, 1487 \\
\hline $\begin{array}{l}\text { Curso(s)/ treinamento(s) de } \\
\text { média/ longa duração } \\
\text { (maiores que } 40 \mathrm{~h} \text { ) }\end{array}$ & 139 & 2,4388 & $-4,913$ & 138 & $*$ &,- 56115 &,- 7870 &,- 3353 \\
\hline Disciplina(s) de graduação & 145 & 2,3448 & $-6,649$ & 144 & $*$ &,- 65517 &,- 8499 &,- 4604 \\
\hline $\begin{array}{l}\text { Disciplina(s) de pós- } \\
\text { graduação }\end{array}$ & 118 & 2,2288 & $-6,814$ & 117 & $*$ &,- 77119 &,- 9953 &,- 5471 \\
\hline Palestra(s) & 184 & 2,3315 & $-10,195$ & 183 & $*$ &,- 66848 &,- 7979 &,- 5391 \\
\hline $\begin{array}{l}\text { Realização de pesquisa(s) } \\
\text { acadêmica(s) }\end{array}$ & 129 & 2,2481 & $-6,901$ & 128 & $*$ &,- 75194 &,- 9675 &,- 5363 \\
\hline $\begin{array}{l}\text { Participação em congressos, } \\
\text { seminários e outros eventos }\end{array}$ & 143 & 2,0769 & $-10,956$ & 142 & $*$ &,- 92308 & $-1,0896$ &,- 7565 \\
\hline Preparação para certificação & 105 & 2,1048 & $-7,095$ & 104 & $*$ &,- 89524 & $-1,1454$ &,- 6450 \\
\hline
\end{tabular}




\subsection{APRENDIZAGEM INFORMAL}

A escala de aprendizagem informal variou entre nenhuma importância (valor 1) e importância muito alta (valor 5). As médias, o valor de $\mathrm{N}$ das variáveis e fatores e os testes $\mathrm{t}$ estão na tabela 4. São destacados em cinza os casos em que a média supera o ponto de corte de forma estatisticamente significativa. Situações em que a média foi estatisticamente inferior ao ponto de corte, ou em que a média não se apresentou como superior ou inferior com significância estatística, não foram consideradas relevantes para a aprendizagem da amostra.

Tabela 4 - Aprendizagem individual informal

\begin{tabular}{|c|c|c|c|c|c|c|c|c|}
\hline & \multirow[t]{2}{*}{$\mathbf{N}$} & \multirow[t]{2}{*}{ Média } & \multirow[t]{2}{*}{$\mathbf{t}$} & \multirow{2}{*}{$\begin{array}{l}\text { Graus } \\
\text { De } \\
\text { lib. }\end{array}$} & \multirow[t]{2}{*}{ sig } & \multirow[t]{2}{*}{$\begin{array}{l}\text { dif. de } \\
\text { média }\end{array}$} & \multicolumn{2}{|c|}{$\begin{array}{l}\text { Int. de conf. de } \\
95 \%\end{array}$} \\
\hline & & & & & & & mínimo & máximo \\
\hline $\begin{array}{l}\text { Autoestudo em livros, revistas, normas e } \\
\text { padrões }\end{array}$ & 216 & 2,9815 &,- 218 & 215 & ,827 &,- 01852 &,- 1857 & ,1486 \\
\hline Autoestudo em sites relacionados ao tema & 225 & 3,3733 & 4,515 & 224 & $*$ & ,37333 & ,2104 &, 5363 \\
\hline Por meio da utilização do software & 254 & 4,1850 & 20,715 & 253 & $*$ & 1,18504 & 1,0724 & 1,2977 \\
\hline Interação direta com superiores hierárquicos & 188 & 2,0266 & 13,647 & 187 & $*$ &,- 97340 & $-1,1141$ &,- 8327 \\
\hline Interação direta com colegas de trabalho & 243 & 3,6626 & 10,727 & 242 & $*$ & ,66255 & ,5409 & ,7842 \\
\hline $\begin{array}{l}\text { Interação direta com profissionais de fora da } \\
\text { empresa }\end{array}$ & 166 & 2,0663 & $11, \overline{5} 10$ & 165 & $*$ &,- 93373 & $-1,0939$ &,- 7736 \\
\hline Interação direta com clientes & 131 & 1,4885 & 22,816 & 130 & $*$ & $-1,51145$ & $-1,6425$ & $-1,3804$ \\
\hline $\begin{array}{l}\text { Interação via software de comunicação } \\
\text { (correio eletrônico, chat, videoconferência, } \\
\text { etc.) com superiores hierárquicos }\end{array}$ & 178 & 1,8596 & 15,195 & 177 & $*$ & $-1,14045$ & $-1,2886$ &,- 9923 \\
\hline $\begin{array}{l}\text { Interação via software de comunicação com } \\
\text { colegas de trabalho }\end{array}$ & 215 & 2,7721 & $-2,872$ & 214 & $* *$ &,- 22791 &,- 3844 &,- 0715 \\
\hline $\begin{array}{l}\text { Interação via software de comunicação com } \\
\text { profissionais de fora da empresa }\end{array}$ & 161 & 1,9193 & 12,789 & 160 & $*$ & $-1,08075$ & $-1,2476$ &,- 9139 \\
\hline $\begin{array}{l}\text { Interação via software de comunicação com } \\
\text { clientes }\end{array}$ & 132 & 1,4394 & $\begin{array}{c}- \\
22,871\end{array}$ & 131 & $*$ & $-1,56061$ & $-1,6956$ & $-1,4256$ \\
\hline
\end{tabular}

As três variáveis de aprendizagem informal mais empregadas dentro da Supde, que apresentaram maior valor de $\mathrm{N}$, foram justamente as três variáveis da aprendizagem informal que foram consideradas significativas de acordo com o teste t: "Autoestudo em sites relacionados ao tema"; "Por meio da utilização do software"; "Interação direta com colegas de trabalho". Ressalva deve ser feita sobre o item "Autoestudo em livros, revistas, normas e padrões", que não está significativamente acima do ponto de corte, mas também não está REAd | Porto Alegre - Vol. 23 - No Especial - Dezembro 2017 - p. 210-231 
significativamente abaixo de 3. Isto significa que a média desta variável é próxima ao ponto de corte, mas que não é possível afirmar que é maior ou menor que o ponto de corte com significância estatística.

O autoestudo por meio de sites online foi importante via pela qual os respondentes adquiriram conhecimento sobre a ferramenta de software livre, o que mostra a importância das iniciativas individuais para aprender, uma vez que o autoestudo é uma prática deliberada dos seres humanos. Esse resultado ilustra os limites, às vezes tênues, entre aprendizagem individual formal e informal. $\mathrm{O}$ autoestudo consiste em prática de aprendizagem informal, mas também em mecanismo de aprendizagem deliberado conscientemente e frequentemente guiado por um método estabelecido, ou melhor, escolhido pelos indivíduos. Tais resultados lançam questionamentos sobre definições já clássicas e amplamente difundidas de aprendizagem informal que estabelecem limites bem rígidos em confronto com aprendizagem formal, conforme Coelho Júnior e Borges-Andrade (2008), Colon (2004) e D'Amelio (2007). O mecanismo irmão, de autoestudo por livros, revistas, normas e padrões, obteve um resultado não significativo possivelmente pelo fato do conhecimento sobre as tecnologias livres não esteja primordialmente armazenado nestas fontes de informação. A informação oferecida pela internet é mais abundante e atualizada, e isso se reflete na diferença entre as modalidades de autoestudo.

A aprendizagem obtida através da prática no uso da ferramenta foi o mecanismo de aprendizagem com maior média, o que é coerente com a literatura de aprendizagem que caracteriza a prática como processo relevante (LOIOLA; PEREIRA; GONDIM, 2011; CAMILLIS; ANTONELLO, 2010; LOIOLA; PORTO, 2008; NERIS, 2005) e importante estratégia comportamental de aprendizagem (PANTOJA; BORGES-ANDRADE, 2009). Um facilitador deste mecanismo de aprendizagem dentro da empresa é a relativa liberdade para se instalar e utilizar softwares livres e a lista de ferramentas livres homologadas pela empresa, que apresenta um bom número de soluções voltadas para o desenvolvimento de sistemas e a realização das tarefas cotidianas dos profissionais.

A interação direta com os colegas se mostrou um mecanismo efetivo de aprendizagem individual, e é indicador de que a comunicação entre os membros da Supde tem colaborado para a aprendizagem dos usuários das ferramentas livres. Também esse resultado, que ressalta a importância da interação em um conjunto específico dos trabalhadores da Supde, corrobora resultados de pesquisas anteriores e revisados no âmbito desta pesquisa (LOIOLA; PEREIRA; GONDIM, 2011; CAMILLIS; ANTONELLO, 2010; SINGH; YOUN; TAN, 2010; 
COELHO JÚNIOR; LOIOLA; PORTO, 2008; CORREIA, 2007; NERIS, 2005). O mecanismo de aprendizagem semelhante ao anterior, relativo à interação com colegas através de software de comunicação obteve um resultado um pouco menos expressivo, possivelmente em virtude da comunicação presencial já suprir boa parte da necessidade, especialmente na comunicação com colegas que trabalhem próximos. Apesar de a empresa disponibilizar softwares para a comunicação interna síncrona e assíncrona, este recurso não foi tão empregado para a aprendizagem em ferramentas livres quanto a interação direta com os colegas.

Os itens considerados de menor significância foram: "Interação direta com profissionais de fora da empresa"; "Interação via software de comunicação com profissionais de fora da empresa"; "Interação direta com superiores hierárquicos"; "Interação direta com profissionais de fora da empresa"; "Interação direta com clientes"; "Interação via software de comunicação (correio eletrônico, chat, videoconferência, etc.) com superiores hierárquicos" e "Interação via software de comunicação com clientes". Diversos fatores contribuíram para que estes mecanismos de aprendizagem individual fossem menos efetivos.

A interação direta com profissionais de fora da empresa foi considerada um fator de menor relevância segundo o critério adotado. Neste mecanismo de aprendizagem os indivíduos empregam sua própria rede social para aprender sobre tecnologias livre aplicadas no ambiente laboral. Esta interação não foi privilegiada pelos respondentes em parte pela falta de oportunidades de congraçamento cotidiano com profissionais de outras empresas que não favorece a construção de uma rede de contatos mais rica por parte dos respondentes. A interação por meio de software de comunicação é também restrita, uma vez que esta comunicação eletrônica é restringida por normas internas da empresa associadas à segurança das informações e certos softwares de comunicação são bloqueados. O fato da empresa pesquisada atuar como concorrente de empresas públicas e privadas na captação de recursos e projetos em licitações públicas é uma situação que desestimula o fortalecimento de redes interorganizacionais, ocasionando impactos nestes mecanismos de aprendizagem.

No caso da interação com os superiores na Supde, a sobrecarga de funções parece impedir uma maior interação entre gestores e subordinados, mas existem vários outros fatores que atuam negativamente sobre esta interação, reduzindo seu impacto como mecanismo de aprendizagem individual. Os líderes das equipes dificilmente podem se aprofundar nas ferramentas a ponto de atingir o nível de conhecimento para transmitir orientações técnicas aos subordinados sobre as tecnologias empregadas, ou têm tempo para fazê-lo. Outra 
possibilidade é o fato de os gestores utilizarem ferramentas diferentes das que são utilizadas pelos desenvolvedores, pela natureza da função desempenhada, muitas vezes desenvolvidas internamente, que não são softwares livres, e sim ferramentas corporativas. Existe ainda a possibilidade do estilo de gestão empregado em algumas equipes estar ancorado em práticas pouco participativas e pouco centradas na interação. Nesse último caso, haveria uma dissonância entre as práticas de gestão adotadas na empresa e de seus gestores e aquelas preconizadas pela literatura em ambientes de trabalho complexos, com reflexos sobre as práticas de aprendizagem individual (ANTONELLO; GODOY, 2009). Nova possibilidade de interpretação pode ser a relativa autonomia com que os pesquisados desenvolvem seu trabalho, dado que é de elevada especialização técnica, o que gera também fortes impactos sobre as práticas de aprendizagem informal, as quais são mais definidas pelos indivíduos que pelas organizações como antes pontuado, mas sofrem condicionantes da forma de organização do trabalho, conforme indicado por Loiola, Pereira e Gondim (2011).

A interação com clientes também não se concretiza mais fortemente como mecanismo de aprendizagem informal, possivelmente pela separação que existe entre a área de desenvolvimento e a área de negócios da empresa, pois esta última é responsável pela interação com os clientes, por meio de uma estrutura profissional, o que corrobora mais uma vez com os resultados de Loiola, Pereira e Gondim (2011). A descentralização geográfica da empresa é um fator complicador da interação física com os clientes, pois o contato com os clientes e o próprio desenvolvimento dos sistemas ocorre com frequência em equipes bastante distantes fisicamente do cliente. Outro fator que fez com que a interação com o cliente tivesse pouca significância é o fato de o cliente apresentar grande conhecimento sobre suas necessidades e sobre o negócio que o software desenvolvido automatiza. Sobre estes itens, o cliente caracteriza-se como fonte de conhecimentos, mas não sobre a tecnologia empregada, seja livre ou não.

\subsection{VARIABILIDADE DOS MECANISMOS DE APRENDIZAGEM INDIVIDUAL}

Analisando-se a perspectiva da variabilidade, a literatura aponta que em vários casos há pouca variabilidade entre os processos de aprendizagem realmente efetivos (LIMA; LIMA; CABRAL, 2013; LOIOLA; PEREIRA; GONDIM, 2011; COOK et al., 2010; LOIOLA; PORTO, 2008; LOIOLA, 2007; NERIS, 2005). Estes ambientes apresentariam restrições em seus processos de aprendizagem, uma vez que resultados apontaram no sentido da associação

REAd | Porto Alegre - Vol. 23 - No Especial - Dezembro 2017 - p. 210-231 
entre maior variabilidade de mecanismos de aprendizagem e maior efetividade da aprendizagem, ocasionada por meio de um maior fluxo de conhecimentos (FIGUEIREDO,2006; CASTRO; FIGUEIREDO, 2005; TACLA; FIGUEIREDO, 2003).

No caso da Supde, foram identificadas três mecanismos de aprendizagem individual que foram considerados estatisticamente significativos, todos de natureza informal, em um conjunto de 20 variáveis: "Autoestudo em sites relacionados ao tema"; "Por meio da utilização do software" e "Interação direta com colegas de trabalho".

A comparação com os resultados da literatura indica baixa variabilidade e conformidade com resultados anteriores (LOIOLA; PEREIRA; GONDIM, 2011; COOK et al., 2010; LOIOLA; PORTO, 2008; LOIOLA, 2007). O estudo de Cook et al. (2010) identificou apenas três mecanismos com mais de $50 \%$ de utilização. Este foi o mesmo número de mecanismos de aprendizagem relevantes encontrados no estudo de Loiola, Pereira e Gondim (2011). Neste estudo, realizado com trabalhadores da agricultura irrigada, foram identificadas apenas opções relativas à aprendizagem informal: "Ajuda de colegas", "Por conta própria" e "Orientação do supervisor" (LOIOLA; PEREIRA; GONDIM, 2011). A literatura aponta para o fato de que existe uma vasta amplitude de mecanismos de aprendizagem, disponíveis aos interessados (LOIOLA; PEREIRA; GONDIM, 2011; COOK et al., 2010).

Outro resultado relevante consiste no fato de que os mecanismos de aprendizagem empregados na Supde indicam autonomia dos respondentes para aprender, uma vez que não existe uma marcante dependência de um instrutor ou condutor do processo.

\subsection{RELAÇÃO ENTRE VARIABILIDADE NA APRENDIZAGEM INDIVIDUAL E CONHECIMENTO OBTIDO}

A relação entre a aprendizagem individual e a variabilidade dos meios empregados na aprendizagem é uma importante questão a ser analisada, que demanda que indicadores confiáveis de variabilidade sejam utilizados. Para investigar o impacto da variabilidade de métodos na aprendizagem obtida pelos respondentes da pesquisa, foi definida uma regra sistemática para extração da variabilidade da aprendizagem de cada indivíduo. Com base nas respostas dos entrevistados, foi feita uma tabulação adicional, atribuindo o valor 1 para cada mecanismo de aprendizagem efetivamente utilizado, e 0 em caso contrário. Com base na soma destes valores, foram criadas três variáveis para cada respondente: Variabilidade da 
aprendizagem individual informal, Variabilidade da aprendizagem individual formal e Variabilidade total dos mecanismos de aprendizagem individual, esta última agregando as duas primeiras.

Para caracterizar associação entre aprendizagem e variabilidade, foi realizado teste correlacional utilizando os métodos de Pearson e Spearman. O resultado mostrou que inexiste associação estatística significativa entre a variabilidade dos mecanismos de aprendizagens formais, informais ou da agregação das duas variáveis, com a aprendizagem efetivamente atingida pelos indivíduos. Foi constatada aprendizagem significativamente estatística no período, mas a mesma não pode ser atribuída a uma maior variabilidade dos mecanismos de aprendizagem individual.

A variabilidade dos mecanismos empregados pelos indivíduos não se reverteu em aprendizagem individual e este resultado contraria a literatura que afirma que níveis mais avançados de aprendizagem tecnológica requerem maior variedade, intensidade de uso e interação entre diferentes mecanismos de aprendizagem, internos e externos às empresas, e formais e informais (FIGUEIREDO, 2006; ROSAL; FIGUEIREDO, 2006; CASTRO; FIGUEIREDO, 2005; TACLA; FIGUEIREDO, 2003), corroborando, no entanto o resultado de outros pesquisadores em pesquisas empíricas (LOIOLA; PEREIRA; GONDIM, 2011; LOIOLA; PORTO, 2008; LOIOLA, 2007; NERIS, 2005). O fato da variabilidade não apresentar o impacto esperado gera novas questões. Esta situação se repete em outros contextos de aprendizagem? Qual o papel e o impacto da variabilidade de opções de mecanismos empregados nos indicadores de aprendizagem?

Estudos adicionais são necessários para individualizar as contribuições da variabilidade, da intensidade e de outras variáveis do ambiente e das tarefas realizadas para a aprendizagem no trabalho, aferindo-se assim o impacto dos métodos empregados para o ganho de conhecimentos. No ambiente da empresa estudada, a eficácia da aprendizagem pode ser mais bem explicada pela maior eficácia dos métodos escolhidos pelos respondentes para aprender, que pela quantidade de opções escolhidas pelos indivíduos. Os resultados obtidos demandam novas investigações em outros contextos visando a elicitação de padrões generalizáveis.

\section{CONSIDERAÇÕES FINAIS}

Pesquisar a variabilidade de mecanismos de aprendizagem é importante, uma vez que REAd | Porto Alegre - Vol. 23 - No Especial - Dezembro 2017 - p. 210-231 
grande esforço é despendido para oferecer modalidades alternativas de aprendizagem individual, sejam do tipo presencial ou não (LAI; KHADDAGE; KNEZEK, 2013; COOK et al., 2010).

A ausência de mecanismos de aprendizagem formal entre os mais significativos é um indicador de que a aprendizagem informal apresenta um apelo mais forte pelo seu dinamismo, foco nas necessidades imediatas e pela autonomia que dá aos aprendizes (CAMILLIS; ANTONELLO, 2010). A predominância da aprendizagem informal também é condizente com o resultado de outras pesquisas sobre aprendizagem (LOIOLA; PEREIRA; GONDIM, 2011; CONLON, 2004). No entanto, mais investigações são necessárias no sentido de clarificar o papel da aprendizagem formal, uma vez que a bibliografia indica que as aprendizagens formais e informais são necessárias (COELHO JÚNIOR; BORGES-ANDRADE, 2008; D'AMELIO, 2007).

A baixa variabilidade dos processos de aprendizagem individual não impediu que a aprendizagem dos indivíduos da amostra fosse comprovada estatisticamente. No caso da empresa investigada, parece haver um ajustamento entre o nível de variabilidade dos mecanismos de aprendizagem individual e as necessidades da empresa. Com autonomia e alternativas de opções de ferramentas para adotar, os empregados aprendem proativamente. Ao mesmo tempo, a empresa evita gastos, uma vez que boa parte das opções escolhidas não apresenta encargos financeiros para a organização. Esse ambiente de experimentação dinâmica de ferramentas apresenta potencial de influência positiva sobre a aprendizagem, e o estudo de suas características consiste em possibilidade a ser explorada em pesquisas futuras.

A inexistência de correlação entre a variabilidade dos mecanismos de aprendizagem e e a obtenção de conhecimentos por parte dos indivíduos faz surgir questionamentos sobre o seu papel e impactos sobre a aprendizagem. Evidencia-se a necessidade de realização de pesquisas qualitativas complementares, para elucidar as razões que fazem certos mecanismos de aprendizagem mais atraentes que outros.

Não é possível generalizar as conclusões deste estudo a outras realidades, uma vez que o mesmo se restringe à realidade de apenas uma empresa em particular. A concentração em apenas uma superintendência de uma empresa pública pode ser vista como limitação, mas também consiste em foco necessário pelas suas características únicas: grande porte; prover serviços para várias outras empresas públicas com os mais variados requisitos; utilização maciça em tecnologia da informação e software livre. O foco em empresa pública, de tecnologia, com software livre, permite a exploração de lacunas no estudo destas áreas 
constatadas na literatura.

Outras possibilidades de investigação compreendem a utilização dos resultados aqui apresentados para análises comparativas, pesquisas longitudinais e para a análise da variabilidade de mecanismos de aprendizagem como variável preditora das aprendizagens individual e organizacional, questão que demanda mais investigação.

\section{REFERÊNCIAS}

ANTONELLO, C. S.; GODOY, A. S. Uma Agenda Brasileira para os Estudos em Aprendizagem Organizacional. RAE - Revista de Administração de Empresas, São Paulo, v. 49, n. 3, p. 266-281, Jul./Set. 2009.

ARAÚJO, M. F. de. Impacto de treinamento e desenvolvimento: uma análise integrada quantitativa e qualitativa. 2005. 250f. Tese (Doutorado em Mestrado em Engenharia de Produção) - Universidade Federal de Santa Catarina - Programa de Pós-Graduação em Engenharia de Produção, Florianópolis.

BELL, S. J.; MENGÜÇ, B.; WIDING II, R. E. Salesperson learning, organizational learning, and retail store performance. Journal of the Academy of Marketing Science, v. 38, n. 2, p. 187-201. 2009.

BULL, G. et al. Connecting informal and formal learning: Experiences in the age of participatory media. Contemporary Issues in Technology and Teacher Education, v. 8, n. 2, p. 100-107, 2008.

CAMILLIS, P. K. de; ANTONELLO, C. S. Um estudo sobre os processos de aprendizagem dos trabalhadores que não exercem função gerencial. RAM - Revista de Administração Mackenzie, v. 11, n. 2, p. 4-42. 2010.

CASTRO, E. C. de; FIGUEIREDO; P. N. Aprendizagem Tecnológica Compensa? Implicações da Acumulação de Competências Tecnológicas parada Acumulação de Competências Tecnológicas para o Aprimoramento de Performance Técnico-econômica em uma Unidade de Aciaria no Brasil. RAC - Revista de Administração Contemporânea. Curitiba: ANPAD, Edição Especial, n. 1, p. 109-133. 2005.

COELHO JÚNIOR, F. A.; BORGES-ANDRADE, J. E. Uso do conceito de aprendizagem em estudos relacionados ao trabalho e organizações. Paidéia, v. 18, n. 40, p. 221-234. 2008. CONLON , T. J. A review of informal learning literature, theory and implications for practice in developing global professional competence. Journal of European Industrial Training, v. 
28, n. 2/3/4, p. 283-295. 2004.

COOK, D. A.; GARSIDE, S.; LEVINSON, A. J.; DUPRAS, D. M.; MONTORI, V. M. What do we mean by web-based learning? A systematic review of the variability of interventions. Medical Education, v. 44, p. 765-774, 2010.

CORREIA, M. P. L. Aprendizagem e Compartilhamento de Conhecimento em

Comunidades Virtuais de Prática: estudo de caso na comunidade virtual de desenvolvimento de software livre Debian-BR-CDD. 2007. 215f. Dissertação (Mestrado em Mestrado em Administração) - Escola de Administração da UFBA, Salvador.

CZERKAWSKI, B. Blending formal and informal learning networks for online learning. The International Review of Research in Open and Distributed Learning, v. 17, n. 3, 2016. D'AMELIO, M. Aprendizagem de Competências Gerenciais - um estudo com gestores de diferentes formações. 2007. 246f. Dissertação (Mestrado em Administração de Empresas) Universidade Presbiteriana Mackenzie - Programa de Pós-Graduação em Administração de Empresas, São Paulo.

ERAUT, M. Informal learning in the workplace. Studies in continuing education, v. 26, n. 2, p. 247-273, 2004.

FIGUEIREDO, P. Capacidade tecnológica e Inovação em Organizações de Serviços Intensivos em Conhecimento: evidências de institutos de pesquisa em tecnologias da Informação e da Comunicação (TICs) no Brasil. Revista de Inovação Brasileira, v. 5, n. 2, p. 403-454. 2006.

HANKE, C. Impacto do Treinamento no Trabalho: Análise de Ações de Capacitação de Auditores do Banco do Brasil. 2006. 118f. Dissertação (Mestrado em Gestão Social e Trabalho) - Faculdade de Economia, Administração, Contabilidade e Ciência da Informação e Documentação - FACE - Programa de Pós-Graduação em Administração - PPGA, Brasília. LAI, K.-W., KHADDAGE, F.; KNEZEK, G. Blending student technology experiences in formal and informal learning. Journal of Computer Assisted Learning. v. 29, p. 414-425. 2013.

LEOPOLDINO, C. B. Avaliação de Riscos em Desenvolvimento de Software. 2004. 151f. Dissertação (Mestrado Acadêmico em Administração) - Universidade Federal do Rio Grande do Sul, Porto Alegre.

LIMA, B. C. C.; LIMA, T. C. B. de; CABRAL, A. C. De A. Estilos de Funcionamento e Mecanismos de Aprendizagem em uma Indústria Criativa de Publicidade e Propaganda. Revista de Carreiras e Pessoas (ReCaPe). ISSN 2237-1427, v. 3, n. 3, 2013. 
LOIOLA, E. Microprocessos de aprendizagem em organizações na Bahia. Relatório de pesquisa-CNPq. Salvador, 2007.

LOIOLA, E.; PEREIRA, M. E.; GONDIM, S. M. G. Aprendizagem e mecanismos de aprendizagem de trabalhadores em empresas da fruticultura irrigada do Baixo Médio São Francisco. Gestão \& Produção (UFSCAR. Impresso), v. 18, p. 91-104, 2011.

LOIOLA, E; PORTO, G. Aprendizagem organizacional das empresas do Prêmio Finep de Inovação. Revista Economia \& Gestão, v. 8, n. 18, p. 32-52. 2008.

\section{LÓPEZ, J. A. C. El Impacto del Aprendizaje en el Rendimiento de las Organizaciones.}

2006. 133f. Dissertação (Mestrado em Administração) - Universidad Nacional de Colombia Sede Manizales - Facultad de Ciencias y Administración, Colômbia.

NERIS, J. S. Microprocessos de Aprendizagem em Organizações do Baixo Médio São Francisco. 2005. 209f. Dissertação (Mestrado em Mestrado em Administração) - Escola de Administração da UFBA, Salvador.

PANTOJA, M. J.; BORGES-ANDRADE, J. E. Estratégias de Aprendizagem no Trabalho em Diferentes Ocupações Profissionais. RAC-Eletrônica, v. 3, n. 1, p. 41-62, Jan./Abr. 2009. RASHMAN, L.; WITHERS, E.; HARTLEY, J. Organizational learning and knowledge in public service organizations: A systematic review of the literature. International Journal of Management Review, v. 11, n. 4, p. 463-494. 2009.

ROCHA, L. A. Software livre e produção colaborativa na internet: um estudo de caso dos instrumentos de comunicação do projeto Gnome. 2007. 81f. Dissertação (Mestrado em Comunicação e Cultura Contemporânea) - Universidade Federal da Bahia - UFBA, Salvador. ROSAL, A. C. L.; FIGUEIREDO, P. N. Aprendizagem Corporativa e Acumulação Tecnológica: a trajetória de uma Empresa de Transmissão de Energia Elétrica no Norte do Brasil. Gestão e Produção. v. 13, n. 1, p. 31-43, jan-abr. 2006.

SIMON, H. A. Bounded Rationality and Organizational Learning. Organizational Science, v. 2, n. 1, p. 125-134. 1991.

SINGH, P. V.; YOUN, N.; TAN, Y. A Hidden Markov Model of Developer Learning Dynamics in Open Source Software Projects. Information Systems Research. 2010. Disponível em: 〈http://ssrn.com/abstract=952608 >. Acesso em 24 Jan. 2015. SOUZA, Y. L. de. A Contribuição do Compartilhamento do Conhecimento para o Gerenciamento de Riscos em Projetos: um estudo na Indústria de Software. 2007. 136f. Dissertação de Mestrado Profissional - Faculdades Integradas de Pedro Leopoldo, Pedro Leopoldo.

REAd | Porto Alegre - Vol. 23 - No Especial - Dezembro 2017 - p. 210-231 
TACLA, C. L.; FIGUEIREDO P. N. Processos de aprendizagem e acumulação de competências tecnológicas: evidências de uma empresa de bens de capital no Brasil. RAC Revista de Administração Contemporânea. Curitiba: ANPAD, v. 3, n. 7, p. 101-126. 2003. WILLE, R. B. Educação musical formal, não formal ou informal: um estudo sobre processos de ensino e aprendizagem musical de adolescentes. Revista da ABEM, v. 13, n. 13, 2005. ZERBINI, T. Avaliação da Transferência de Treinamento em Curso à Distância. 2007. 321f. Tese (Doutorado em Psicologia) - Universidade de Brasília - UNB, Brasília. 